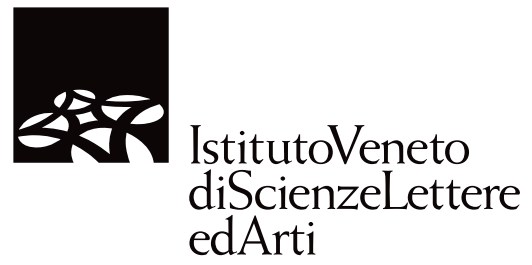



ISTITUTO VENETO DI SCIENZE, LETTERE ED ARTI

\title{
IL COMMONWEALTH VENEZIANO TRA 1204 E LA FINE DELLA REPUBBLICA IDENTITÀ E PECULIARITÀ
}

\author{
a cura di
}

GHERARDO ORTALLI, OLIVER JENS SCHMITT, ERMANNO ORLANDO 
ISBN 978-88-95996-52-3

Il volume riporta le relazioni presentate al Convegno

Il "Commonwealth" veneziano tra 1204 e la fine della Repubblica.

Identità e peculiarità

The Venetian "Commonwealth" between 1204 and the End of the Republic. Identity and Specificities

(Venezia, 6-9 marzo 2013)

promosso da:

Istituto Veneto di Scienze, Lettere ed Arti,

Österreichische Akademie der Wissenschaften

VISCOM (Visions of Community), Austrian Science Fund FWF

Regione del Veneto

Università Ca’ Foscari

Centro Tedesco di Studi Veneziani

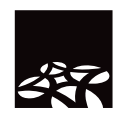

IstitutoVeneto

diScienzeLettere edArti
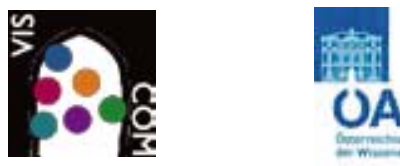

\section{OAW}

Università

Ca'Foscari 0 REGIONE on VENETO

Venezia

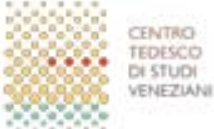

Progetto e redazione editoriale: RugGeRo Rugolo

(C) Copyright Istituto Veneto di Scienze, Lettere ed Arti - Venezia

30124 Venezia - Campo S. Stefano 2945

Tel. 0412407711 - Telefax 0415210598

ivsla@istitutoveneto.it

www.istitutoveneto.it 


\section{INDICE}

\section{Parte I \\ LA DIMENSIONE DIACRONICA. \\ LO STATO VENEZIANO TRA PREMESSE MEDIEVALI \\ E DERIVE TARDO MODERNE}

Gherardo Ortalli, The Genesis of a Unique form of Statehood, Between the Middle Ages and the Modern Age . . . . . . . Pag. 3

Gian Maria Varanini, I nuovi orizzonti della Terraferma . . " " 13

Monique O'Connell, The Contractual Nature of the Venetian State .................... " 57

David Jacoby, The Expansion of Venetian Government in the Eastern Mediterranean until the late Thirteenth Century. . . . " 73

\section{Parte II \\ LO STATO IN FUNZIONE. STRUTTURE DI COMANDO E GOVERNO}

Luciano Pezzolo, La costituzione fiscale dello Stato venezia-

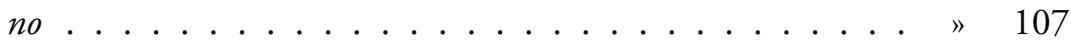

Andrea Zannini, Una burocrazia repubblicana. Stato e amministrazione a Venezia tra XVI e XVIII secolo . . . . . . . . » 131

Benjamin Arbel, Una chiave di lettura dello Stato da mar veneziano nell'Età moderna: la situazione coloniale . . . . . . . " 155 
PARTE III

LO STATO IN FUNZIONE.

COMUNICAZIONE, TRATTATIVE E LEGITTIMAZIONE

Egidio Ivetic, Territori di confine (secoli XV-XVIII) . . . . . Pag. 181

Oliver Jens Schmitt, «Altre Venezie» nella Dalmazia tardomedievale? Un approccio microstorico alle comunità socio-politiche sullisola di Curzola/Korčula . . . . . . . . . . . . . . " 203

Alessandra RizzI, Dominante e dominati: strumenti giuridici nell'esperienza 'statuale' veneziana . . . . . . . . . . . " 235

Nikolaos Karapidakis, Dominants et dominés dans le Levant vénitien: les zones d'ombre des identités . . . . . . . . . . . " 273

PARTE IV

LO STATO DEBOLE. FRA MARE E TERRA

Guillaume Saint-Guillain, Protéger ou dominer? Venise et la

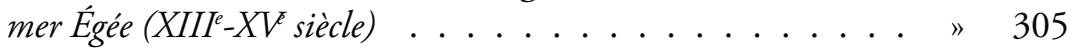

Thierry Ganchou, Sujets grecs crétois de la Sérénissime à Constantinople à la veille de 1453 (Iôannès Tortzélos et Nikolaos Pôlos): une ascension sociale brutalement interrompue . . " 339

Serghei Karpov, Colonie o capisaldi. Verso Tana, Trebisonda e il Mar Nero, secc. XIV-XV . . . . . . . . . . . . . . . . . " 391

Ermanno Orlando, Mobilità, migrazioni, intrecci . . . . . » 405 


\section{Parte V \\ SPAZI DI MOVIMENTO, CONFRONTO E CONTROLLO. DENTRO E FUORI I CONFINI}

Paolo Preto, "Causar la morte di questo tristo" fa «un gran

bene alla nostra patria» . . . . . . . . . . . . . . . Pag. 433

Eric Dursteler, "Portare San Marco nel cuore» Strategie di integrazione all'interno della nazione veneziana a Istanbul . . . " 455

Piero Del Negro, L'esercito e le milizie . . . . . . . . . . . " 473

Indice dei nomi . . . . . . . . . . . . . . . " 495

Elenco dei relatori . . . . . . . . . . . . . . . " 525 



\section{Oliver Jens Schmitt}

\section{“ALTRE VENEZIE" NELLA DALMAZIA TARDO-MEDIEVALE? UN APPROCCIO MICROSTORICO ALLE COMUNITÀ SOCIO-POLITICHE SULL'ISOLA DI CURZOLA/KORČULA ${ }^{1}$}

Il carattere e le peculiarità della statualità veneziana sono discussi nei contributi di questo volume soprattutto con approcci strutturali e/o spaziali che privilegiano quasi esclusivamente una prospettiva dall'alto. I grandi concetti - Repubblica aristocratica o stato patrizio, stato composito, impero o Commonwealth come propone il titolo del nostro volume sono ormai ben noti nella discussione specializzata ${ }^{2}$.

Negli ultimi anni l'attenzione a pratiche extra-istituzionali, di mediazione e negoziazione del potere, allo stato debole, alle periferie e alle loro interazioni con il centro sono emersi come temi centrali del dibattito storiografico; è stato criticata giustamente anche l'attenzione esagerata alla violenza o in genere ai casi di conflitto, di natura

${ }^{1}$ Questo saggio si inserisce nell'ambito del progetto "Visions of community" (VISCOM) della Fondazione di ricerca scientifica austriaca FWF.

${ }^{2}$ G.M. VARAnini, La terraferma veneziana nel Quattrocento e le tendenze recenti della storiografia consultato sul sito http://www.academia.edu/4230487/Varanini_La_ terraferma_veneziana_nel_Quattrocento_e_le_tendenze_recenti_della_storiografia; J.E. Law, The Venetian Mainland State in the Fifteenth Century, "Transactions of the Royal Historical Society», VII/2 (1992), pp. 153-174; M. O'Connell, Men of Empire. Power and negotiation in Venice's Maritime State, Baltimore 2009; L'amministrazione della giustizia penale nella Repubblica di Venezia (secoli XVI-XVIII), II, Retoriche, stereotipi, prassi, a cura di G. ChIodi - C. Povolo, Verona 2004; I. LaZZarini, L'talia degli stati territoriali. Secoli XIII-XV, Roma-Bari 2003; Origini dello Stato. Processi di formazione statale in Italia fra medioevo ed età moderna, a cura di G. Chittolini - A. Molho - P. SCHIERA, Bologna 1994; una presentazione descrittiva dell'amministrazione veneziana nella parte prevalentemente ortodossa dello Stato da mar è offerta da Anastasia PAPADIA-

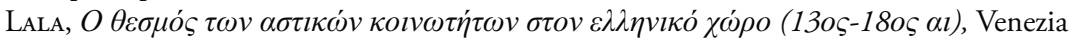
2004. Per una recente prosepttiva generale sullo Stato da mar vd. B. Arbel, Venice's Maritime Empire in the Early Modern Period, in A Companion to Venetian History, 14001797, a cura di E.R. Dursteler, Leiden-Boston 2013, pp. 125-253. 
eccezionale, e che non rappresentano l'insieme assai oscillante delle varie forme di presenza e di dominio veneziano nelle sue molteplici espressioni nel Dogado, nella Terraferma e nello Stato da mar. Il punto cardine della maggior parte di queste discussioni teoriche è il diritto o meglio i vari sistemi di diritto esistenti e applicati nel vasto dominio veneziano tra Bergamo e Cipro, il sistema giuridico che fu sottoposto a numerosi cambiamenti lungo i secoli della dominazione veneziana ${ }^{3}$. Riassumendo una ricchissima bibliografia, ci si riferisce in questa sede soltanto a due recenti lavori di Ermanno Orlando, la sua monografia sul Dogado e un saggio importante dedicato al sistema giudiziario nella Dalmazia quattrocentesca che discute in dettaglio i risultati della recente rivalutazione della statualità veneziana in una prospettiva comparativa che tiene conto del contesto italiano ${ }^{4}$.

Era proprio nell'ambito di questa discussione che già negli anni ottanta studiosi come Michael Knapton proposero una visone d'insieme che intendeva sormontare la frammentazione dell'analisi dello stato veneziano in almeno tre spazi/ambiti, rappresentando vere e proprie sottodiscipline della venezianistica (Venezia città, la Terraferma e lo Stato da mar), di cui la scarsa comunicazione è stata ripetutamente lamentata ${ }^{5}$. Nella sua recente monografia sul Dogado due e trecente-

${ }^{3}$ E. Orlando, Alla ricerca della statualità medievale, «Le carte e la storia. Rivista di storia delle istituzioni», XV/1 (2009), pp. 107-115.

${ }^{4}$ ID., Altre Venezie. Il Dogado veneziano nei secoli XIII e XIV (giurisdizione, territorio, giustizia e amministrazione), Venezia 2008; ID., Politica del diritto, amministrazione, giustizia. Venezia e la Dalmazia nel basso medioevo, in Venezia e Dalmazia, a cura di U. Israel - O.J. Schmitt, Roma 2013, pp. 9-61. Cfr. anche E. Ivetic, Venezia e l'Adriatico orientale: connotazioni di un rapporto (secoli XIV-XVIII), in Balcani occidentali, Adriatico e Venezia fra XIII e XVIII secolo, a cura di G. Ortalli - O.J. Schmitt, Venezia-Vienna 2009, pp. 239-260.

5 G. Cozzi - M. Knapton, La Repubblica di Venezia nell'età moderna. Dalla guerra di Chioggia al 1517, Torino 1986; M. Knapton, Venice and the Terraferma, in The Italian Renaissance State, a cura di A. GAMBERINI - I. LAZZARINI, Cambridge 2012, pp. 132-155. Questo volume dimostra le difficoltà della definizione spaziale della storia veneziana. Gli editori hanno imposto al contributo di Knapton un concetto che limita Venezia allo Stato da Terra e esclude lo Stato da Mar. Dall'altra parte viene integrato nell'ambito spaziale di una storia dell'Italia rinascimentale il Tirolo del Sud; Marco Bellabarba a cui gli editori hanno dato l'incarico di presentare The Feudal Principalities: the East (Trent, Bressanonel Brixen, Aquileia, Tyrol and Gorizia), pp. 197-219, oscilla tra frontiere «naturali» e la 
sco Ermanno Orlando sviluppa il concetto di "Altre Venezie», concetto proposto da Gherardo Ortalli, e dimostra i complessi rapporti orizzontali e verticali tra il centro e la periferia nascente, nascente perché Orlando insiste sulla dinamica dei rapporti che si accelerò nel secondo Trecento ${ }^{6}$. Il modello sviluppato per il Dogado, l'idea di altre Venezie, ben inteso tenendo conto delle differenze culturali e politiche tra il Dogado e il nostro caso, meriterebbero di essere applicati anche al caso della Dalmazia quattrocentesca. E questo vale anche per una dimensione della statualità veneziana che è strettamente connessa con il modello di "Altre Venezie», cioè l'importanza dei patti di dedizione, del rispetto per gli statuti e le consuetudini locali, e della funzione mediatrice del dominio veneziano, un modello che già da più di ormai trent'anni struttura le analisi della Terraferma sotto il dominio veneziano ${ }^{7}$. Questo saggio propone di combinare i modelli dell'ampia discussione, accennata qui solo in pochissime righe, con un approccio che finora è stato scelto solo raramente nel contesto della storiografia sullo Stato da mar, cioè la prospettiva microstorica sulle grandi strutture e sviluppi nello stato veneziano del tardo medioevo.

Quest'approccio non propone di sostituire i modelli d'interpretazione già esistenti, ma di completarli offrendo una prospettiva che illustri e approfondisca i nostri concetti della presenza statuale veneziana oltremare, nel nostro caso l'isola di Curzola (Korčula) nella Dalmazia meridionale ${ }^{8}$. Non si può certo negare la tensione che nasce tra un

discussione critica di concetti nazionalisti otto e novecenteschi. L'integrazione del Tirolo nella storia italiana medievale e l'esclusione dello Stato da mar veneziano riflettono comunque un disequlibrio nella concezione spaziale del volume. Una discussione della dimensione spaziale dello «Stato italiano nel Rinascimento» manca, e questa mancanza provoca incoerenze nell'analisi del soggetto.

${ }^{6}$ Orlando, Altre Venezie.

7 Tra i numerosi lavori di Gherardo OrTalli cfr. Entrar nel Dominio: le dedizioni delle città alla Repubblica Serenissima, in Società, economia, istituzioni. Elementi per la conoscenza della Republica Veneta, I, Istituzioni ed economia, Verona 2002, pp. 49-62; Le modalità di un passaggio: il Friuli occidentale e il dominio veneziano, in Il Quattrocento nel Friuli occidentale, pp. 13-33; Il ruolo degli statuti tra autonomie e dipendenze: Curzola e il dominio veneziano, «Rivista storica italiana», XCVIII/1 (1986), pp. 195-220; cfr. anche la collana Corpus statutario delle Venezie, a cura di G. Ortalli.

${ }^{8}$ Per un altro esempio di insularità veneziana cfr. G. SAINT-Guillain, Amorgos au XIVe siècle. Une seigneurie insulaire entre Cyclades féodales et Crète vénitienne, «Byzantinische 
programma teoretico vasto, cioè quello di analizzare l'intero complesso territoriale dello stato veneziano - e lo studio di un caso quasi microscopico. L'approccio microstorico collega la descrizione della dimensione concreta, antropologica con grandi temi e strutture, senza però ridurre la complessità delle interazioni tra i due livelli dell'analisi a una semplice dipendenza degli individui dalle strutture politiche, socio-economiche e culturali. La concentrazione sul livello personale, quasi biografico, di persone appartenenti alla società locale, nel caso di Curzola, di fronte ai rappresentanti dell'amministrazione veneziana è giustificata in buona misura da un argomento pratico di grande rilevanza: l'esistenza di un archivio locale la cui ricchezza oltrepassa la maggior parte degli archivi 'provinciali' dello stato veneziano nel Quattrocento. L'archivio tardomedievale di Curzola, oggi conservato nell'Archivio di Stato di Zara, costituisce il raro esempio di un archivio locale quasi completo, un archivio che comprende per il Quattrocento - a partire del 1420, anno della dedizione - un penale e un civile molto ricco, il notarile, una parte della corrispondenza dei rettori con le autorità centrali a Venezia, le contralittere ossia registri delle navi in partenza dal porto, gli atti del Consiglio di Curzola, fino agli atti di guardiani di campo che riferivano ogni mattina al conte i danni causati da animali penetrati in campi e giardini. La «dimensione umana» (G.M. Varanini) dell'amministrazione veneziana si rivela in tutte le sue varie e colorate dimensioni, in una sfumatura che non s'incontra quasi mai altrove'. L'esempio curzolano va quindi al di là di una semplice storia locale. Il caso microscopico di una piccola isola contribuisce alla discussione teoretica sul carattere dello stato veneziano; e l'approccio microstorico all'isola microscopica approfondisce le possibilità metodologiche che ci offre la documentazione locale. Le possibilità di studiare i rapporti e le interazioni tra 'governanti' e 'governati' ${ }^{10} \mathrm{o}$

Zeitschrift», 94/1 (2001), pp. 62-189; ID., Îles grecques au temps de l'Empire latin. Andros et Lemnos au XIIIe siècle, "Mélanges de l'École française de Rome. Moyen âge», 113/1 (2001), pp. 579-620; ID., Seigneuries insulaires: les Cyclades au temps de la domination latine (XIII'-XV' siècle), «Médiévales», 47 (2004), pp. 31-46.

9 Varanini, La Terraferma, p. 18.

${ }^{10}$ N.E. Karapidakis, I rapporti fra "governanti e governati" nella Creta veneziana: una questione che può esserer riaperta, in Venezia e Creta, a cura di G. Ortalli, Venezia 1998, pp. 233-244. Importanti sono i saggi di B. ArbeL, Résistance ou collaboration? Les 
tra rappresentanti dello stato e della società di Venezia e i vari strati sociali curzolani si rivelano indubbiamente come eccezionali. Lo studio del lavoro concreto dell'amministrazione, programma delineato da Michael Knapton ormai un quarto di secolo fa, si lascia realizzare grazie a questa documentazione densa ${ }^{11}$. Partendo da un caso microscopico, l'isola di Curzola e alcuni protagonisti della sua vita politica e sociale nei primi decenni del dominio veneziano, si propone una nuova prospettiva sul funzionamento dello Stato da mar in una zona in cui la "vicinanza fisica alla capitale» ${ }^{12}$ si faceva sentire grazie a reti di comunicazione rapidi e regolari. La possibilità di rintracciare le biografie di contadini e altri individui "semplici» non ci deve fuorviare troppo sino a perderci o nel «infiniment petit» (R.J. Loenertz) o in un biografismo individualista che trascura il contesto socio-culturale e soprattutto la fitta rete di legami tra il Comune, le comunità e gruppi più fluidi che esistevano o si formavano a Curzola. Una chiave di lettura per le strutture sociali a Curzola rimangono gli Statuti, il testo principale non solo per la ricerca moderna, ma il punto di riferimento cardinale della società curzolana nel Quattrocento ${ }^{13}$. Troppo spesso questo testo è stato letto e interpretato in maniera statica: la divisione della popolazione in patrizi e popolani e i conflitti che ne derivavano invitavano molti storici della Dalmazia medievale a una lettura che operava con categorie di una lotta di classe che rivelava più (come nella Jugoslavia titina) o meno direttamente l'influsso del pensiero marxista. Inoltre, considerata alla luce del conflitto classico tra detentori di potere e privilegi politici e il ceto che ne era escluso, la società curzolana risultava strutturata in modo più complesso. Il tradizionale modello binario patrizi-popolani, città potente-contado sottomesso è

Chypriotes sous la domination vénitienne, in ID., Cyprus, the Franks and Venice, 13th-16th Centuries, Aldershot 2000, parte n. VIII, e Entre mythe et histoire: la légende noire de la

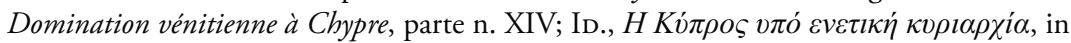

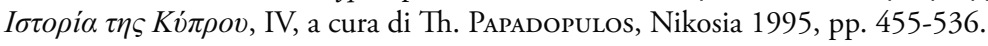

${ }^{11}$ M. Knapton, Le Istituzioni Centrali per l'amministrazione e il controllo della Terraferma, in Venezia e le Istituzioni di Terraferma, Bergamo 1988, p. 44.

12 Knapton, Le Istituzioni Centrali, p. 42.

13 Ortalli, Il ruolo degli statuti; Gli accordi con Curzola, a cura di E. Orlando, Roma 2002; Zbornik radova Znanstvenog skupa Statut grada i otoka Korčule iz 1214. godine, a cura di Z. ŠEPAROVIĆ, Zagreb 1989. 
contraddetto dalle realtà sociali di un'isola ove molti patrizi resiedevano nei villaggi, ove la città non era capace di imporre la sua volontà politica su un contado con forti tradizioni di autoamministrazione istituzionalizzata, dove l'orgoglio politico delle comunità rurali si nutriva della coscienza e della conoscenza degli Statuti, del diritto, della buona educazione almeno dei leader contadini, dell'accesso al sapere che offrivano le linee di comunicazione marittime. Consapevoli delle proprie tradizioni giuridiche i contadini ricorrevano abilmente alle possibilità di comunicazione, di appello nella capitale previste nel sistema amministrativo veneziano. Il Comune e l'isola di Curzola erano dunque strutturati secondo categorie definite negli Statuti, ma comunità meno formali che trascendevano spesso i limiti costituzionali esercitavano un influsso spesso più importante sulle pratiche di potere sull' isol $\mathrm{a}^{14}$. Queste comunità erano formate sulla base di parentele, di interessi politici ed economici, ma anche di sapere, di convinzioni 'ideologiche' (spesso anche locali) e, aspetto importante, di emozioni come segno di appartenenza. Frazioni, gruppi d'interesse e 'cliques' costituiscono l'oggetto di una viva discussione nella storiografia italiana degli ultimi anni, una discussione che insiste sulla fluidità delle appartenenze, sui rapidi cambiamenti di lealtà e sui processi di formazione dei gruppi e sul loro carattere spesso effimero, sempre in relazione con la loro funzione politica e socio-economica che è sottoposta alle trasformazioni delle costellazioni sociali e politiche ${ }^{15}$. Questi modelli interessano la Dalmazia medievale, anche se, come nel nostro caso, bisogna ricordare le particolarità locali (precauzione metodologica normale in questo tipo di studi); e queste particolarità si rivelano nella mancanza di frazioni che caratterizzano i grandi Comuni dell'Italia settentrionale nell'epoca tardo-medievale. Utili e importanti sono osservazioni sull'uso retorico e politico delle categorie costituzionali (patrizio, popolani, statuti) e di grandi idee politiche nella negoziazione di potere ${ }^{16}$. Nel caso curzolano l'integrazione dell'auto-immagine

${ }^{14}$ Cf. E. MuIr, The Idea of Community in Renaissance Italy, "Renaissance Quarterly», 55/ (2002), pp. 1-18.

${ }_{15}$ M. Gentile, Factions and parties: problems and perspectives, in GamberiniLaZZARInI, The Italian Renaissance State, pp. 304-322, vd. pp. 307-309.

${ }^{16}$ Cfr. O’Connell, Men of Empire, pp. 97-118. 
di Venezia ('il mito') nel pensiero locale - integrazione maturata in lunghi secoli di amministrazione o vicinanza con Venezia - può essere considerata un processo chiave. Questo discorso presente nei «capitula» o petizioni di altri possedimenti veneziani d'Oltremare penetrava, come vedremo, fin nel linguaggio quotidiano dei Curzolani che l'adattavano ai propri usi e bisogni ${ }^{17}$. Questo nuovo elemento discorsivo riflette il lento cambiamento delle strutture. Infatti, il caso curzolano ci offre la possibilità di studiare la connessione tra due sviluppi di mutazione: il difficile e lento processo di integrazione amministrativa dell'isola nello stato veneziano e la trasformazione del pensiero politico e della retorica politica della società locale. Curzola entrò nello Stato da mar con strutture costituzionali e sociali tipiche del modello comunale adriatico che trovavano la più marcata espressione nella consapevolezza politico-sociale degli appartenenti ai vari gruppi del Comune e alle comunità, una tradizione di partecipazione politica e di 'vecchio diritto'. In questa sede basta ricordare elementi essenziali del quadro costituzionale che determinano le pratiche socio-culturali dei nostri protagonisti ${ }^{18}$ : la dedizione dell'isola nel 1420 , dedizione negoziata dai patrizi; la scissione interna della società insulare non solo in patrizi e popolani come altrove in Dalmazia, ma anche la frammentazione interna dei popolani in "cittadini antichi»" ${ }^{19}$, «villici», e soprattutto la tradizione di autonomia politica nello spazio rurale che si esprimeva nei «veće» ossia congregazioni: queste regolari congregazioni dei villici, consigli di villaggio, pretesa di rappresentazione esterna dei contadini; ma anche il fatto che molti patrizi erano residenti in campagna e non in città e partecipavano anche ai consigli di villag-

17 Gentile, Factions and parties, p. 317; O.J. Sснмitt, Das venezianische Südosteuropa als Kommunikationsraum 1400-1600, in Balcani occidentali, Adriatico e Venezia fra XIII e XVIII secolo/Der westliche Balkan, der Adriaraum und Venedig, 13.-18. Jahrhundert, a cura di G. Ortalli - O.J. Schmitt, Vienna-Venezia 2009, pp. 77-101; Arbel, Venice's Maritime Empire, pp. 187-188.

${ }^{18}$ Cf. O.J. Sснмiтt, Korčula sous la domination de Venise au XVe siècle. Pouvoir, économie et vie quotidienne dans une île dalmate au Moyen Âge tardif, Paris 2011 (Les conférences du Collège de France) (e-book http://conferences-cdf.revues.org/).

19 DrŽavni arhiv u Zadru. Arhiv Korčule (=DAZ AK), 10/3/1, «povolo de la cità». 
gio $^{20}$. Dal punto di vista tipologico, Curzola è da situare tra i comuni classici della terraferma con la città dominante e il suo contado e il caso eccezionale di Brazza (Brač), isola senza città, senza centro politico, società insulare composta di villaggii ${ }^{21}$. Su Curzola la prima fase del dominio veneziano è caratterizzata dall'aspra lite tra città e villaggi sul potere politico, lite che si cristallizzava nella questione a chi appartenesse il privilegio di interpretare gli statuti. Importa anche schizzare la ripartizione del peso demografico; nel 1451 una lista dei contribuenti al salario del medico curzolano ci offre un primo indizio sul numero di abitanti, 198 nomi per Curzola-porto, 73 per il villaggio di Žrnovo, 46 a Čara, 54 a Smokvica. Occorre ricordare che la lista non è esaustiva non includendo poveri e vedove, l'unica donna che appare sulla lista è l'amante di uno dei nostri protagonisti ${ }^{22}$.

Queste osservazioni servono da primo e rapido schizzo del contesto strutturale nel quale si svolgono le vicende dei protagonisti della nostra microstoria. L'approccio microstorico è diviso in due parti. Nella prima parte si narra la storia dei fratelli Çanin e Çuanin Dragačić provenienti dal villaggio curzolano di Čara (ca. 1410 - ca. 1475), due contadini che percorsero un'ascesa socio-economica - ma anche di prestigio - in buona misura dovuta alla loro rapida e abile integrazione e adattamento al modello politico e ideologico dello stato veneziano $^{23}$. Una seconda parte contestualizza le vicende dei fratelli presentando casi di negoziazione e di conflitto nei primi decenni del

${ }^{20}$ «omnes nobiles de Zara (il villaggio di Čara su Curzola) et populares fecerunt consilium»; DAZ AK, 6/6/6, f. 7v del 1425.

${ }^{21}$ T. Raukar, Srednjovjekovni Brač na razmedu društva, in ID., Studije o Dalmaciji u srednjem vijeku, Split 2007, pp. 357-367; ID., Komunalna drušrva u Dalmacji u XIV stoljeću, «Historijski zbornik», 33-34 (1980-1981), pp. 138-209; ID., Komunalna društva u Dalmaciji u XV st. i u prvoj polovini XVI stoljeća, «Historijski zbornik», 35 (1982), pp. 43-118; J. VRANDEČić, Značajke društveno-gospodarskog razvoja Brača za vrijeme mletačke uprave (1420-1797), «Historijski zbornik», 46/1 (1993), pp. 67-88; A. Cvitanić, Iz dalmatinske pravne povijesti, Split 2002, pp. 407-466.

${ }^{22}$ DAZ AK, 13/23/8, ff. 111r-115r.

23 Per la storia di questi due personaggi vd. O.J. Sснмітт, Storie d'amore, storie di potere: la tormentata integrazione dell' isola di Curzola nello Stato da mar in una prospettiva microstorica, in Venezia e Dalmazia, pp. 89-109, ID., Micro-history and Lebenswelten as approaches to late mediveval Dalmatian history: a case study of Korčula, in Spomenica akademika Sime Cirkovića, a cura di S. Rudić, Belgrado 2011, pp. 137-158. 
dominio veneziano. Benché i due fratelli siano i protagonisti naturali del nostro approccio, ricordiamo che al centro di ogni discussione sulla statualità si trovano il diritto e le istituzioni, cioè, a Curzola come anche in tutta la Dalmazia o la Terraferma, i patti di dedizione e gli statuti locali ${ }^{24}$. Mentre nella storiografia italiana si osserva un ritorno delle istituzioni nella discussione su comunità e statualità ${ }^{25}$, nel caso della Dalmazia le istituzioni hanno da sempre prevalso come categoria di analisi. Il caso curzolano deve tener conto di ambedue gli sviluppi storiografici: bisogna insistere su un approccio più sottile e flessibile nell'analisi delle strutture amministrative, del quadro costituzionale e istituzionale, cioè nel senso del dibattito italiano e internazionale degli ultimi trenta anni; dall'altra parte sembra opportuno evitare possibili esagerazioni di un'interpretazione che sottovaluti il peso delle istituzioni, un peso che si riflette anche nel discorso quotidiano, e non solo in quello ufficiale e retorico riferito a situazioni di negoziazione e di rappresentazione.

Il punto di partenza di un'analisi microstorica è la scelta di un individuo e di un gruppo ristretto di individui sulle cui tracce viene descritto un contesto socio-culturale. La nostra scelta cade sui due fratelli quasi inseparabili, anche di nome. Çanin e Çuanin Dragačić dal villaggio curzolano di Čara possono essere accompagnati lungo un arco cronologico di più di cinquanta anni, e la loro storia finisce bene: malgrado tanti conflitti e risse ambedue muoiono di morte naturale. La mancanza di un grande processo che di solito costituisce il corpus fondamentale degli studi microstorici è più che equilibrata dalla varietà dei processi e dei testi che ci permettono di ricostruire lo sviluppo delle strategie politiche e retoriche dei nostri protagonisti e la nascita di un'identità modellata su strutture socio-politiche, prima di tutto giuridiche e costituzionali locali e veneziane ${ }^{26}$. Mentre la concentra-

${ }_{24}$ R.C. Mueller, Aspects of Venetian Sovereignty in Medieval and Renaissance Dalmatia, in Quattrocento Adriatico, a cura di C. Dempsey, Bologna 1996, pp. 29-56.

${ }_{25}$ Gentile, Factions and parties, p. 310.

${ }^{26}$ C. Ginzburg, Hexensabbat. Entzifferung einer nächtlichen Geschichte, Berlin 2005; N. Zemon Davis, Die wahrhaftige Geschichte von der Wiederkehr des Martin Guerre, Berlin 2004; A. LüDKE, Alltagsgeschichte, Mikro-Historie, historische Anthropologie, in Geschichte. Ein Grundkurs, a cura di H.-J. GoerTz, Reinbek bei Hamburg 2001, pp. 547-578; per un'analisi di processi nello Stato da mar vd. A. Viggiano, Tra Venezia e 
zione su un caso unico riflette anche un momento ben preciso, che è in un certo senso anche statico, il nostro studio di un caso invece offre tutta la dinamica di un mezzo secolo di dominio veneziano su una isola dalmata, cioè dai primi passi dei conti veneziani dopo il 1420 fino all'inizio della minaccia ottomana (1468 ss.) che cambiò radicalmente i rapporti tra Curzolani e la Serenissima, una cesura da paragonare alla rottura nel Dogado provocata dalle guerre nella seconda metà del XIV secolo $^{27}$. Un corpus di testi che non si limiti a processi, ma che includa anche il notarile evita anche il pericolo metodologico di privilegiare momenti di scontro e di violenza; questi danno, certo, rilievo al ruolo dei fratelli nella società curzolana e nei loro rapporti con le autorità veneziane. Il loro ruolo però non può essere ridotto a una violenza troppo presente in un certo tipo di fonti ${ }^{28}$.

Raccontiamo in poche parole le vicende biografiche dei nostri protagonisti. Çuanin e Çanin Dragačić, originari del villaggio di Čara nell'entroterra dell' isola, sono nati verso il 1410; appaiono per la prima volta nel 1425 nei registri della cancelleria veneziana come testimoni in un processo; sappiamo anche che il loro padre morì nel $1434^{29}$. Figli di un contadino semplice di cultura croata (appare due volte nei pochissimi casi in cui la cancelleria registra insulti in lingua croata, e non in traduzione ${ }^{30}$ ), i fratelli quasi omonimi attirano il nostro interesse tredici anni più tardi quando difesero in un processo il diritto di forestieri, cioè di Ragusani, e sedici anni più tardi, quando si misero alla testa di una sollevazione contadina contro l'aumento delle tasse. Al 1439 data il loro conflitto con vari membri del ceto

Creta. Conflittualità giudiziarie, identità sociali e memorie familiari nello Stato da mar del Quattrocento, in Venezia e Creta, pp. 106-149.

27 Per la storia preveneziana di Curzola vd. S. Doкоza, Dinamika otočnog prostora, Split 2009, per il periodo veneziano Sснмітт, Korčula sous la domination de Venise au XVe siècle.

${ }^{28}$ Cfr. il volume Streitkulturen. Gewalt, Konflikt und Kommunikatio in der ländlichen Gesellschaft (16.-19. Jahrhundert), a cura di M. ERIKsON - B. KRUG-Richter, ColognaWeimar-Wien 2003, in particolare il contributo di P. Wettmann-Jungblut, Gewalt und Gegen-Gewalt. Gewalthandeln, Alkoholkonsum und die Dynamik von Konflikten anhand eines Fallbeispiels aus dem frühneuzeitlichen Schwarzwald, pp. 17-58.

${ }^{29}$ DAZ AK, 6/6/6, ff. 42r, 43r, 173r (Zaninus Franetich, Zaninus filius Francisci Dragacich).

${ }^{30}$ Ibid., f. 129v («Lages caco pas», «Menti come un cane»), f. 134v. 
nobile, conflitto che accompagna i due fratelli fino alla morte: Çanin morendo nel 1458, Çuanin venti anni dopo. Tra le tappe importanti della loro biografia di guerriglia continua con i patrizi menzioniamo soltanto la ribellione contadina del 1441 , il processo montato contro Franuša, l'amante di Çuanin, processo cassato da Francesco Foscari nel 1449 a causa della manipolazione da parte del conte e del partito patrizio, la resistenza dei fratelli, con la spada in mano, contro i giudici patrizi e varie risse nelle calli e sulla piazza di Curzola ${ }^{31}$. Questa storia di violenza comunque si accompagna in pari grado all'ascesa socioeconomica dei fratelli, che compravano terre, bestiame, divennero dazieri, commercianti di vino e di sale, spesso anche in compagnia con patrizi, accumularono procure, stabilirono una rete di amicizie e di lealtà, si trasferirono in città dove figurarono tra i personaggi di spicco, e soprattutto viaggiarono spesso nella Dominante in qualità di ambasciatori e/o uomini d'affare. Nella vita politica eccelsero non solo come rappresentanti locali dei contadini, ma anche come ambasciatori ufficiali dei popolani e poi anche dell'isola («steti a Veniesia avanti el conspeto dela Nostra Serenissima Signoria in beneficio de tuta quela università de popolo de Curzola per ambasador lor mexi 3... litigando con ser Forte et ser Francisco de Obradi zoè cum li ambasadori deli zentilhomeni» ${ }^{32}$ ). I Dragačić appaiono in decine se non centinaia di casi nei registri della cancelleria nelle loro qualità di proprietari, commercianti o procuratori; in questa sede basta fare una piccola sosta ogni dieci anni, cioè nel 1438, 1448, 1458 e 1468 per seguire la dinamica del rapporto tra $\mathrm{i}$ fratelli e le strutture veneziane locali e centrali. I giovani fratelli appaiono ad esempio nel 1438 come testimoni nell'atto di vendita di una vigna (10 giugno 1438), denunciano i guasti in una loro vigna (8 luglio 1438), vendono un pezzo di "terra aratoria» (8 ottobre 1438), accettano una procura nel caso di un' eredità $(9 \text { ottobre })^{33}$; nel gennaio 1440 trovano tre vacche nella loro vigna a Vela luka ${ }^{34}$, e già nel 1437 fanno parte di una commissione locale "ad

31 Vd. M. O'Connell, The Sexual Politics of Empire: Civic Honor and Official Crime outside Renaissance Venice, "Journal of Early Modern History», 15 (2011), pp. 1-18.

${ }^{32}$ DAZ AK, 10/14/4, f. 1r.

33 Ibid., 8/11/1, ff. 168v, 172r, 179r, 189v.

${ }^{34}$ Ibid., 7/5/6, f. $14 \mathrm{r}$ 
dessignandum animalia ${ }^{35}$. Dieci anni più tardi, nel 1448 agiscono da «fideiussores» di un patrizio che avevano già rappresentato a Venezia nel commercio di panni; si trovano in qualità di dazieri dell'ufficio del sale a Curzola in stretti legami con il clan patrizio degli Obradović, sono registrati nella cancelleria come procuratori del muratore Ratko Juračić e degli uomini dei villaggi dell'isola, sono attivi nel commercio di cereali con un commerciante abruzzese, noleggiano una nave per trasportare sale a Sebenico, chiedono al villaggio di Žrnovo il resto del salario per la loro ambasciata a Venezia ${ }^{36}$. Vent'anni dopo le tensioni con i patrizi, Zuanin, rimasto solo dopo la morte di Zanin che fece il suo testamento il 28 luglio 1456, difende gli interessi del clero locale contro il vescovo veneziano assai prepotente, Luca Leon ${ }^{37}$, interpretando una bolla papale (1 febbraio 1460). Infine nel 1468, Dragačić continua ad accumulare procure, sostiene la causa del guardiano dei francescani sul cosiddetto scopulo, rappresenta il conte in un processo contro un patrizio ${ }^{38}$, denuncia la vedova di suo fratello (che l'aveva maritata "contra la mia voluntà») ${ }^{39}$. Non mancano nemmeno le piccole risse, ma sono ora i figli di Zuanin, Francesco e una figlia, a esserne coinvolti: nel settembre del 1468 , i due ragazzi sono aggrediti dai guardiani di campo durante la vendemmia, «la mia fiolla [...] caschò cum la faza in terra [...] e bate mio fiol» ${ }^{40}$.

Il percorso biografico dei fratelli è certo non del tutto eccezionale; offre l'esempio di un membro dell'élite locale benestante come la troviamo anche altrove; uomini che sapevano far carriera avvicinandosi sempre di più al ceto amministrativo veneziano e alle strutture di potere del Dominio veneziano. Il fascino del nostro caso deriva dalla dimensione quasi intellettuale dei protagonisti che parlano, attaccano e si difendono davanti ai rettori e di fronte alle autorità centrali e che sanno sviluppare quello che possiamo chiamare un pensiero politico. Infatti, il figlio di un modesto contadino diventò un abile interprete

${ }^{35}$ Ibid., 7/9/1, f. 96v.

${ }^{36}$ Ibid., 9/2/2, ff. 3r, 7r, 10v-11r, 50r, 51r, 54r, 63r.

37 Ibid., 15/28/6, f. 178r.

38 Ibid., 20/36/9, f. 1r, 41r, 42v, 43r; 20/36/10 f.1v.

39 Ibid., 20/36/12, f. 3r.

40 Ibid., 20/36/12, f. 12v-13r. 
del diritto locale, un negoziatore esperto, un giurisperito che con una bolla papale in mano difese gli interessi di un prete locale davanti al vescovo.

Il processo d'integrazione nel mondo politico, amministrativo e discorsivo di Venezia non si svolse però senza frizioni. Infatti, soprattutto nei primi anni del dominio veneziano, i Dragačić svolgevano le loro attività politiche dentro e fuori il sistema costituzionale - almeno dal punto di vista dei patrizi e dei conti: dentro, perché utilizzavano i «capitula» cioè le petizioni indirizzate alle autorità centrali previste $e$ incoraggiate dalla Serenissima; fuori, perché radunavano i contadini nelle tradizionali congregazioni di villaggio, cosa che agli occhi dei patrizi costituiva una flagrante violazione degli statuti e un caso di ribellione, agli occhi dei conti veneziani ora un atto di disobbedienza, ora un'espressione politica ammessa o almeno tollerata dei rappresentanti della Serenissima. Il diritto di adunarsi opponeva, all'inizio degli anni quaranta, patrizi e contadini, mentre i conti veneziani si muovevano incerti affinché il Senato ponesse fine a questa tradizione che i Dragačići descrivevano in dettaglio, citando tutti i casi accaduti nella fase iniziale del dominio veneto:

nui de populo sempre avimo posudo far la congregacion nostra anchor anci che fossemo soto la nostra e quanto magis adesso che l'a voludo e vol la dita Nostra Illustrissima Signoria per lo suo Consilio de Pregadi che posiamo far dagando noticia ali Retori o in avanti over da puo facta congregacion [...] lo qual privilegio e tuti altri sono stadi confirmadi per lo dito Consilio de Pregadi, offerandomi provar che infina quando vene la armada de Puiesi asidiar questa terra, nui de populo fesemo nostro conselio in la ecclesia de San Piero e molte altre volte mente che nui se rezevemo per li nostri zentilomeni. Item dapoi l'avimo fato al tempo de misser Pangrati Zorzi. Item in tempo de miser Tomaso Michel, item in tempo de miser Marco Gradenigo, item ancora nel tempo dela Magnificencia Vostra, e sempre facemo abiando bisogno in queli caxi neli qual ni conceduto la Nostra Illustrissima Signoria sichè me offero provar che quela adunanza non è sta alguna novità, ma nostra antiga usanza e consueto ${ }^{41}$.

\footnotetext{
${ }^{41}$ Ibid., 10/14/4, f. 167r.
} 
Mentre per i fratelli e i loro partigiani, le adunanze erano pienamente legali e le accuse del conte e dei patrizi quindi senza fondamento costituzionale, i patrizi invece si lamentavano di «questa arogancia deli chavi $[. .$.$] ", "gridando per le piace cum seguito de vilani multi$ si'an deffidado le persone voler combater mitando la terra a rumor quasi ogni çorno vituperando e digando vilania ali çudixi e zentilomini nostri, fazando secte e congregaciun non fo mai per Dio»; «quisto è segno di una pessima semença, magnifico misser lo Conte, obviati alli schandali $»^{42}$. Si nota la violenza del linguaggio emozionato che esprime anche la paura dei patrizi di fronte a una corrente politicorurale molto forte; si osserva anche la tattica dei patrizi di strumentalizzare il conte per placare conflitti locali. Benché non abbiano mai apertamente contestato la legittimità del governo veneziano, i partiti socio-politici sull'isola impararono ben presto a distinguere tra lo stato veneziano - cioè le istituzioni centrali e l'idea dello stato - e i suoi rappresentanti, cioè il conte, che diventava oggetto di critica o addirittura di attacchi seri; questo vale anche per i Dragačići che proclamarono nel 1442 «El non avea tanta força miser lo Conte e li çudixi ponere me in carceribus ${ }^{43}$. Nei primi due decenni di dominio veneziano, $\mathrm{i}$ rapporti dei fratelli con i conti erano abbastanza tesi; questi ultimi si lasciavano troppo spesso sedurre dal ceto patrizio e trascuravano l'obbligo di rimanere imparziali di fronte ai conflitti locali. Le minacce dei fratelli indirizzate ai conti invece non erano percepite automaticamente dalle autorità centrali come atto di insubordinazione $\mathrm{e}$ di ribellione. È importantissimo sottolineare che i conti e le autorità centrali non perseguivano sempre la stessa politica. Quando, come accadeva spesso, un conte si lasciava ingannare da un partito locale, i concorrenti si rivolgevano direttamente alle autorità centrali, talvolta due ambasciate, una nobile e una popolana, partivano per Venezia ${ }^{44}$. Così iniziò il contatto a livello politico con l'amministrazione veneziana che si sviluppò rapidamente al di là dalla rappresentanza locale del dominio veneto e coinvolse ben presto anche le autorità centrali. Era proprio la comunicazione diretta con queste ultime che provocò

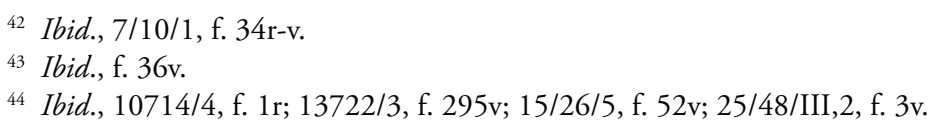


un conflitto gravissimo tra il conte e i patrizi da un canto, i popolani dall'altro, quest'ultimi, rappresentati dai fratelli Dragačić che difesero le loro congregazioni e il diritto di inviare indipendentemente dai patrizi le loro ambasciate a Venezia ${ }^{45}$. I fratelli contestavano il monopolio patrizio di comunicazione con le autorità centrali, e queste ultime sostenevano la posizione dei fratelli per creare un equilibrio politico su una isola politicamente divisa.

Difendere il proprio diritto era anche una scuola di retorica. Purtroppo non sappiamo niente sull'educazione dei fratelli, ma è evidente che fecero progressi impressionanti nell'arte di presentare la propria posizione politica e giuridica. Ascoltiamo uno dei fratelli argomentare davanti al conte (si tratta di una risposta alla denuncia di un patrizio a causa dell'esportazione di vino a Venezia):

respondo che Zugno de questa parte non dixe punto la verità ni may proverà cum verità zoe testimonii legitimi idonei over degni de fede, salvo s'el non fosseno alchuni mei inimicissimi li quali si metino instessi avanti a testificar contra de mi, zo che non credi che la Vostra summa Iustitia vorà comportar per non esser justo nè honesto quando havereti intexo le mie opposition... Magnifico misser lo Conte, io non fezi may alchuna piezaria a Zugno per lo patron ni promessa, e se la ho fato, deverià parer nota in cancelleria chome se sol far in fato de piezarie ${ }^{46}$.

Occorre rilevare la familiarità con i meccanismi della cancelleria veneziana, e con le sue procedure formali, e, come vediamo subito, anche con le finezze della legge locale. Invocando «la lege di Curzula confermada per la Nostra Glorioxa Signoria de Venexia» uno dei fratelli ricorda abilmente la sua fedeltà alla Serenissima. Segue una lunghissima dissertazione dotta sui testimoni negli statuti dell' isola, con citazione di vari paragrafi, ad esempio:

altra lege sta a capitolo 16 la qual dixe che alchuno testimonio non debia esser agetà s'el non serà sta chiamato saltem per una dele parte

45 Ibid., 7/9/1-2, f. 167v.

46 Ibid., 17, fascicolo Processus doni Radetini cum Marco Marsich simul cum aliis processibus, f. $34 \mathrm{r}$. 
per testimonio specialiter" ovvero "concludo che non pò vi dir esser tolto per testimonio contra di me, perché nostro Statuto a capitulo 36, altro a capitulo 37 non vol che inimicho manifesto sia tolto per testimonio ${ }^{47}$.

Questa intima conoscenza degli Statuti non era eccezionale a Curzola; al contrario, era abbastanza diffusa sull' isola in tutti i ceti sociali. Nell'arco cronologico stupisce il percorso intellettuale dei fratelli: i giovani oratori popolari, aggressivi e volgari si tramutarono lentamente negli esperti di finezze giuridiche degli anni cinquanta e sessanta.

Mentre i primi scontri dei fratelli con Venezia incarnata nella persona del conte furono assai violenti, i Dragačići presto affrontarono anche altre dimensioni della statualità veneziana, anzitutto quando si recarono a Venezia, prima come ambasciatori che negoziarono con il Senato, e poi per appellarsi alla corte degli Auditori novi. Alla fine davanti ai Pregadi cercarono di ottenere, ma invano, la legalizzazione delle congregazioni contadine "che posiamo far dagando noticia ali Rectori o in avanti o dapuo facta congregacion $»^{48}$. Al mantenimento del loro consiglio era legato strettamente il privilegio di inviare ambasciate indipendentemente dalle ambasciate dei patrizi, ciò che dimostra che i Dragačići avevano capito bene l'importanza della comunicazione istituzionalizzata, cioè i cosidetti capitula «che nela nostra congregacion possiamo ordenar la nostra imbasada per contradir a chusì tale loro iniqui leze» ${ }^{49}$; abilmente cercavano di giustificare le adunanze dei villaggi con l'argomento che quelle servivano all'unico scopo di garantire la rappresentanza dei loro interessi nel quadro costituzionale imposto dalla Serenissima. "Me ho appellato viva voce ai Signori Aldidori novi dele sententie [...] perché io intendo perseguir la mia appellation ${ }^{50}$ sono le parole di Zuanin quando, nel 1448, si opponeva alla sua condanna a Curzola. Questo suo intervento dimostra anche fino a che grado si erano familiarizzati con i meccanismi della giustizia veneta. Avevano imparato a distinguere tra il conte e le autorità centrali, e a utilizzare la comunicazione con la capitale per

\footnotetext{
47 Ibid., f. 38r.

${ }^{48}$ Ibid., 10/14/4, f. 166v.

${ }^{49}$ Ibid., f. $167 \mathrm{v}$.

${ }^{50}$ Ibid., 12/20/1, f. $8 \mathrm{v}$.
} 
indebolire gli avversari locali, conte e patrizi. Il conte Pietro Soranzo ad esempio venne qualificato "non chome conte e rector, ma chome parte adversa e chome advocato» che «levosse suxi e comenzò rengar e avocar contro de mi e rengò un gran pezo digando che mi meritava punimento o ogni mal», "cosa de uno rector mai alo mondo non fo vista ni aldida, che zudexi davanti el qual se dumanda raxon e justitia avocarsi per la parte ${ }^{51}$. La cassazione della condanna montata fu certamente un momento decisivo nell'atteggiamento dei Dragačići di fronte a Venezia: delusi da un conte, furono riconfortati nella capitale: la ducale di Francesco Foscari costituì il simbolo del loro trionfo politico contro gli avversari nobili.

E benché lo stesso doge avesse finalmente interdetto le congregazioni contadine, i Dragačići divennero seguaci fedeli della Repubblica di San Marco, e il discorso critico o aggressivo di fronte ai conti venne meno per sempre. Nel caso dei Dragačić, la funzione mediatrice del conte inizialmente fallì, perché l'elemento atto a riparare le tensioni locali era costituito dalle istituzioni centrali che assolvevano al loro ruolo solo grazie alla rapida integrazione dei fratelli curzolani. Una volta superato il conflitto personale con il conte grazie al sostegno delle autorità centrali, i Dragačić svilupparono un vero desiderio di portare le loro querele davanti alle istituzioni competenti nella capitale, e allo stesso tempo cresceva la familiarità con le procedure della giustizia veneta e la produzione di documenti e testimonianze. Nei Dragačić, il sistema veneto trovò strenui addetti della propaganda statale e dei vantaggi che si offrivano a seguaci fedeli. Da parte loro, i Dragačić capirono quanto era utile adattarsi alle esigenze della retorica statale di Venezia. Abbiamo già visto come abbozzavano l'immagine di un conte imparziale e giusto per opporsi nella capitale agli intrighi del conte Soranzo. Venezia, "la fontana della giustizia» ${ }^{52}$ era il contrario della "Turchia over in luogo dove non se tien ni raxon ne justitia alchuna» ${ }^{53}$. Dragačić sapeva far l'elogio della giustizia veneziana, "la vostra santa justitia», per presentarsi come «servidor vostro soto le ale e protetion

\footnotetext{
51 Ibid., 12/20/1, s.p.

52 Ibid., 10/14/4, s.p. (f. 1r).

${ }^{53}$ Ibid., 17/32/3, f. 21v.
} 
vivo" ${ }^{54}$. Allo stesso tempo impararono anche a maneggiare l'arma della denuncia: riuscirono ad allontanare il loro nemico giurato, il medico Giorgio Grubšić, che mai aveva celato la sua opposizione contro il dominio veneto. Quando questi commise l'imprudenza di insultare in pubblico un uomo che esaltava una vittoria navale sul Regno di Napoli, aggiungendo che un'alleanza tra Napoli e l'Ungheria avrebbe distrutto il dominio veneziano in Dalmazia, i suoi giorni a Curzola furono contati ${ }^{55}$.

D’altro canto, gli avversari del Dragačić non esitavano neanche a denunciare il leader dei contadini quando questi apparve in una posizione poco favorevole; nel febbraio del 1456 Dragačić aveva impedito sulla piazza di Curzola l'arresto di un suo seguace, e questo incidente offrì ai patrizi sedenti nella loggia l'occasione di dipingerlo davanti ai giudici come nemico dell'ordine che avrebbe giurato di assassinare il conte.

Inutile aggiungere che anche i patrizi ricorrevano alle formule del discorso veneziano sulla giustizia. Il Dragačić completava la sua immagine di suddito leale presentandosi come vittima d'intrighi e calunnie dei patrizi, della loro violenza che avrebbe indotto una patrizia a proclamare "che le manzava lire una de le mie carne ruste chi le dessi ${ }^{56}$. Egli, insisteva, «mi habiando stà contro questi zintilhomeni tante volte a Venecia» dove riuscì a mettere fine al monopolio nobiliare dell'elezione dei conti; e siccome aveva provato l'atteggiamento poco leale di alcuni nobili, anche questo suo atto avrebbe contribuito a stabilizzare il dominio veneto. L'ascesa politico-sociale dei Dragačić si svolgeva in un sistema di potere formato dal conte, dalle autorità centrali, dai patrizi e dai popolani di Curzola. Malgrado la collaborazione puntuale e opportunistica con certi patrizi, la differenza sociale costruita dagli Statuti rimase intatta - le comunità socio-costituzionali dei patrizi e dei popolani che funzionano anche come comunità

${ }^{54}$ Ibid., f. 22r.

55 Ibid., 13/22/3, f. 366r-v: «comenzono a parlar de la guera che fo rota fra la nostra Signoria e Rè de Ragona e l'a dito maistro Zore have a dir e dixe a miser Pasqual in bona fe: in malora la signoria ha pigliato guera cum re e Ragona perché s'el Re de Ragona farà liga cum Vaivoda Ivanis de Ungaria per Dio piglarà tuta Dalmatia e Istria, el corpo de Veniexia».

56 Ibid., 17/32/3, f. 30r. 
di conflitto e di fiducia ${ }^{57}$ si percepivano l'una l'altra come avversari e concorrenti. Ambedue cercavano di rinforzare la propria posizione con l'appoggio delle due emanazioni della statualità veneziana, le autorità centrali e il conte locale. Il successo socio-politico dei fratelli è strettamente legato alla capacità di comprendere e utilizzare i meccanismi dello stato veneziano e il 'mito', cioè l'auto-immagine di Venezia. È fuori dubbio che la tradizione locale di auto-rappresentazione, di autonomia contadina, di litigi sull'interpretazione degli Statuti - che sopravvivevano proprio nelle teste dei Curzolani, statuti che non erano un codice, un pezzo di carta, ma una personalità astratta, straordinariamente viva in tutti i ceti sociali - aveva profondamente segnato i fratelli. I Dragačić impararono a combinare questa eredità locale (e adriatica) alle esigenze e offerte del nuovo Dominio ${ }^{58}$. Tale mobilità - intellettuale e spaziale - permise loro di farne un uso perfetto. Sarebbe comunque erroneo isolare il caso dei fratelli dalla struttura sociale dell' isola. Infatti, l'appartenenza alla comunità politico-sociale dei contadini, soprattutto alla comunità di villaggio, serviva da appoggio principale. Queste comunità erano multifunzionali; garantivano non solo certi diritti e privilegi, offrivano non solo protezione nel caso di urgenza, ma costituivano vere e proprie comunità emotive formate da legami personali, di amicizia, di parentela, soprattutto di solidarietà incondizionata in caso di conflitto. Una parola equivoca, un insulto contro gli uomini che avevano difeso l'interesse dei contadini a Venezia bastavano per mobilitare queste emozioni di solidarietà. La loggia di Curzola era spesso il teatro di spontanei confronti tra popolani e patrizi, ed entrambe le parti si sottraevano al controllo dei rappresentanti di Venezia. Il potenziale di mobilitazione sociale rimaneva alto in ambedue le comunità, dei patrizi e dei popolani. Questi confronti aumentavano il prestigio sociale

${ }^{57} \mathrm{Vd}$. Muir, The Idea of Community, pp. 4-6.

${ }^{58}$ Lo stesso Dragačić espone in un processo nel 1462: «digo che la dita lege si è donà valida et autenticha per esser confermada ut supra e messa in volumen Statuti laqual lege sta a capitoli over a carte 2 in lo Statuto" (DAZ AK, 17, Processus doni Marci Radetini); nel 1464 invoca «nostra lege civil et municipal zoe Statuti nostri de Curzula confermadi per la Nostra Ill.ma Dugal Signoria de Veniexia» e "chomo vol anche una nostra lege in volume nono de Statuti laqual dixe e vol che ad ogni cossa possano buttar dui over tre testimoni idonei» (ibid., 18/33/16, ff. 202r, 244r). 
dei fratelli; e siccome vi ricorrevano spesso, rafforzavano ogni volta la loro posizione e il loro capitale simbolico.

Tutto questo percorso biografico è strettamente connesso con la stabilizzazione del dominio veneto nell'isola. Ora bisogna allargare la prospettiva dell'analisi e chiedersi quanto eccezionale o normale fosse stato il caso dei fratelli nel contesto socio-culturale di Curzola nel primo Quattrocento. Il maggior evento nella storia dell'isola, i patti con Venezia e la dedizione, seguiti da un periodo difficile, aggravato da tensioni politiche e continui conflitti sull'interpretazione degli statuti, aveva segnato la giovinezza dei fratelli. Ma la tradizione giuridica con la quale avevano familiarizzato per prima erano stati il diritto curzolano, soprattutto la sua applicazione nello spazio rurale, e certamente anche le consuetudini locali, comprese quelle dei pastori, nel loro villaggio di origine, cioè Čara. All'inizio della dominazione veneziana, negli anni venti del XV secolo, il conte veneziano sedeva sul banco di giustizia («in bancho lapideo contiguo cum domo ser Johannis Angeli iuxta plateam comunis ${ }^{59}$ ) affiancato da tre giudici locali, patrizi potenti e orgogliosi del diritto di poter scegliere il conte, patrizi che controllavano strettamente l'interpretazione degli statuti da parte del conte e che non esitavano a limitare il suo spazio d'azione facendo sempre riferimento ai patti ed agli statuti. Aveva un peso particolare la tradizione delle congregazioni rurali. Nel luglio 1425 «tutti di Çara e i popolani fecero un consiglio e inviarono alcuni uomini per espellere i detti animali e i pastori ${ }^{60}$; i conflitti tra contadini, le comunità rurali da un canto, e patrizi e i loro pastori dall'altro erano numerosi. Le pretese del ceto dominante di Curzola-città erano respinte con forza dalle istituzioni contadine che non celavano il loro orgoglio politico, un orgoglio basato sul diritto di aggregarsi indipendentemente dal patriziato urbano.

I Dragačić vivevano anche un inizio tormentato del dominio veneziano. I rettori veneziani si confrontavano non solo con numerosi conflitti tra comunità locali, ma anche con diversi livelli di critica assai aperta e franca. Benché nessuno osasse mettere in dubbio la legittimità del dominio veneziano, diventò evidente che almeno una parte dei

\footnotetext{
59 Ibid., 6/1/6, f. 2 r.

${ }^{60} \quad$ Ibid., 6/6/6, f. $7 \mathrm{v}$.
} 
patrizi che avevano negoziato il patto di dedizione vedevano nella presenza di un rettore veneziano una formalità che non cambiava le strutture di potere sull'isola. Nel settembre del 1428 i giudici patrizi litigavano con il conte sulle modalità del suo pagamento. «Respondentes nos iudices quod dominus comes omnia potest tamquam comes et dominus et sumus parati ad omnem eius obedientiam [...] nos non sumus petentes tantum quantum sit nostra comunitas et quia comunitas elegit ipsum et non nos tres iudices in singularitate» ${ }^{61}$. Come definiscono i giudici il potere veneziano, la relazione tra dominio veneziano e istituzioni locali? Il conte è sì domino supremo, ma è eletto dal Comune. L'entità astratta è la comunitas locale, i cui diritti sono difesi dai tre giudici, che insistono sul fatto che rappresentano un Comune politico e che solo questo Comune è fonte di legittimo potere. Di fronte al Comune si trova il conte che è percepito come immagine o personalizzazione del potere veneziano. I giudici non utilizzano concetti astratti per descrivere il carattere del dominio veneziano. Che ciò non significhi che modelli meno concreti non circolassero nell'élite curzolana viene illustrata da un altro esempio.

La tensione tra patrizi curzolani e il conte esplose in un momento di crisi. Nell'autunno 1428, la peste apparve nel porto, e molti patrizi si rifugiarono nei villaggi. Il rettore organizzò un'amministrazione improvvisata ricorrendo al sostegno di coloro che non possedevano case sicure in campagna, popolani e soprattutto forestieri, da Sebenico, dalla Narenta (Neretva), da Ragusa, da Stagno (Ston) e da Murano. Quando il cancelliere veneziano costrinse un patrizio che voleva tornare in città a rispettare il bando generale, quello, ser Criaco Luxe, insultò il cancelliere ( $« \mathrm{Ha}$, ha magistro, io tene incaco sulla tua barba di merda») e minacciò di bombardare la città e di metterla a fuoco. Infatti, entrò, la notte seguente, in città con un gruppo di bravi reclutati in un villaggio vicino ${ }^{62}$. Il processo contro questo caso di disobbedienza contiene tutta una serie di discorsi sul carattere e la legittimità del potere sull'isola. Il nobile ribelle distingueva tra 'noi' e 'voi', e per lui, gli 'altri' erano prima di tutto i forestieri: «nolumus forenses» è il grido di guerra, i forenses essendo Italiani e Dalmati, soprattutto Ragusani.

\footnotetext{
${ }^{61}$ Ibid., ff. 144r-145r.

${ }^{62}$ Ibid., ff.146r-147r.
} 
«nolumus hic forenses, sufficientes cum sumus nos terrigeni nostram defendere civitatem». I terrigeni come termine di comunità: ser Criaco fece appello ai sentimenti campanilistici (contro il grande vicino Dubrovnik); i terrigeni sono quelli che contribuiscono alla difesa della città e perciò godono dei diritti di cittadinanza. Che il rettore avesse chiamato i forestieri alla difesa, minacciava gravemente questo concetto di appartenenza e di comunità. La delimitazione tra "terrigeni» e «forestieri» non si limita a immigranti senza diritti politici. «Tu se forestier vegnudo non so donde» - questo rimprovero è indirizzato al cancelliere veneziano. Questi risponde:

$\mathrm{O}$ ser Criace. Tu michi iniurias absque causa et dicis quod me incacas, sed cave tibi, ne cum tua superbia te ipsum incacas. Et dicis quod ego non permito te in tuam civitatem intrare. Civitas non est tua, ymo est nostre Serenissime Ducalis Dominationis, et non ego, sed ordo factus per dominum comitem et officiales pro salute omnium [...] est qui non permictitis intrare ${ }^{63}$.

Il rappresentante del dominio veneziano difende la legittimità del potere della Dominante; nello stesso tempo dimostra la sua consapevolezza della dimensione simbolica e politica del conflitto. A chi appartiene la città? All'élite tradizionale o alla Signoria? È evidente che due concetti di comunità e di statualità si incontrano: lo stato basato sulla comunità dei terrigeni, un gruppo con il suo proprio diritto e una forte coscienza di potere che significa più concretamente: possesso del territorio; e una statualità di Dominazione astratta, cioè non una comunità di persone, una Dominazione che agisce per il bene comune, "pro salute omnium». Un concetto astratto e uno scopo supremo ugualmente astratto.

Lo scambio di argomenti e insulti raggiunge il culmine quando il nobile attacca direttamente la persona del rettore: «tu es tirannus et vis tiranare», "ego me ulciscar de te». La risposta del conte: "Civitas non est tua, sed est Serenissime Dominationis, et ego non sum tiranus, sed sum comes, sed si tiranus fuero, hoc habet conoscere dominatio no-

\footnotetext{
${ }^{63}$ Ibid., f. 147r-v.
} 
stra, sed tu es tiranus qui non vis legibus et ordinibus subiacere" ${ }^{64}$. La dominazione veneziana come tirannia. Il pensiero politico a Curzola era familiare con concetti della teoria di stato. Il nobile e il rettore discutono sulle basi dello stato e della statualità. La tirannia non conosce regole e è sinonimo di potere arbitrario. Il 'mito' veneziano risiede nel diritto e nella stabilità politica, si basa su "pace e giustizia», sulla garanzia del vecchio diritto. Il patrizio contesta il carattere legittimo dell'amministrazione veneziana, perché ai suoi occhi il conte aveva violato il diritto locale. La risposta del conte è rimarchevole: ripete che la città non appartiene al vecchio ceto dominante, ma allo stato veneziano. Questo stato è un'entità astratta, dotata di leggi e ordini cui sono sottomessi tutti, i sudditi e i rappresentanti dello stato. «Non sono tiranno, ma sono conte»: la prima parte della risposta riflette l'orgoglio personale del patrizio veneziano; la seconda parte della frase invece rimanda al sistema politico di Venezia, uno stato che, secondo il conte, non tollera la tirannia e dispone di meccanismi per punire i tiranni. Lo stato appare in questo dialogo in due ipostasi: come potere concreto sulla città e come rappresentazione di principi supremi di giustizia e garante del buon governo.

L'auto-immagine di Venezia propagata dai suoi rappresentanti su Curzola si diffondeva anche negli strati popolani. Nell'aprile del 1428 un popolano replicò a un patrizio che si era vantato: «Scavi nostri e suditi nostri, nui podimo far ogni cosa in contra di vui qual che ne par in quela» con le parole: «Tu non è conte et tu no i tuta Corzula, tu non a tu razon a dir si farà vilania di poveri homini perché non sono vostri scavi né vostri suditi, sono scavi e suditi dela Excelsa Signoria de Venezia la qual Dio mantegna in bon stado [...] siando sutu el dominio de la Excelsa Signoria de Venezia la qual non dà tortizar nisuna povera persona contra di razon $»^{65}$. Il popolano definisce la sua visione del buon governo. Lo stato veneziano garantisce uguaglianza davanti al diritto e combatte il potere arbitrario dei patrizi. Essere sotto il

${ }^{64} \mathrm{Ibid}$., f. 149 r. Per il problema dell'abuso di potere nello Stato da mar cfr. G. SaInT-GuIllain, Abus de pouvoir ou gouvernance de proximité? Administrateurs vénitiens et administrés à travers l'exemple de la prise d'Argos par les Ottomans en 1397, in Venetia-Argos. Simadia tis venetikis parusias sto Argos kai stin periochi tu, a cura di Ch. Maltezu - A. Panopulu, Athina-Venetia 2010, pp. 81-119.

${ }^{65}$ DAZ AK, 6/6/6, f. 127r. 
dominio protetto da Dio significa che l'amministrazione si basa sulla «razon», principio opposto all'orgoglio dei nobili che vedevano nei popolani solo «schiavi e sudditi». Lo stato veneziano e i suoi principi supremi alimentavano una strategia identitaria che strumentalizza l'idea di stato propagandata da Venezia come argomento nei conflitti locali invocando la propria lealtà, insistendo sul fatto di aver accettato non solo il dominio, ma anche il suo spirito - tutto quello costituisce un'arma pericolosa. «Vos Nobiles facitis malas consuetudines, destruitis insulam ${ }^{66}$, questa frase riassume una seconda posizione importante dei popolani. La violazione del diritto - garantito da Venezia - da parte dei patrizi rovina l'equilibrio politico-sociale nell'isola.

Così, nei primi anni della presenza veneziana a Curzola, diventa visibile tutta la fragilità della posizione del conte pochi anni dopo il massiccio intervento della flotta veneziana nella Dalmazia centrale e meridionale; si aggiungono altri casi degli anni $1426-28$ per dimostrare che non si tratta di eccezioni: il 27 settembre 1426, il conte, benché fosse ricorso ai pugni, non riuscì a riscuotere da un contadino il dazio del vino e si rivolse ai circostanti con le parole «vedete come si tratta qui il vostro conte»; è particolarmente significativo come il conte descrive il suo linguaggio del corpo: «maxima cum paciencia nullum faciens movimentum» di fronte alla resistenza di un contadino. Quest'immobilità non esprime tanto la dignità del rettore, ma costituisce piuttosto un contrasto all'uso di violenza alla quale era ricorso prima. Infatti, il conte aveva battuto il contadino e l'aveva tirato per i capelli. Benché questi non osasse aggredire fisicamente la persona del conte, quest'ultimo si trovò finalmente in una situazione di debolezza non essendo riuscito a far valere la sua autorità. Per non perdere la faccia, si ritrasse in un linguaggio opposto che dovrebbe esprimere la superiorità e la dignità della sua persona ${ }^{67}$. Il 5 ottobre 1427 , un altro popolano minacciò il conte: "Conte, conte, tu te pentirai et non fai ben ${ }^{68}$. Neanche i patrizi esitavano a criticare la corte: ser Giovanni Angeli accusò il conte e il giudice Giovanno Prvošić di «fare

\footnotetext{
${ }^{66}$ Ibid., 6/1/6, f. 3r.

${ }^{67}$ Ibid., 6/6/6, f. 87 r.

${ }_{68}$ Ibid., f. $91 \mathrm{r}$.
} 
tirannia ${ }^{69}$. Altre volte non celavano affatto il disprezzo per il sistema giuridico «tu mentiris [...] sicut canis et nisi essemus coram comite, ego tibi mitterem cultellam in pectorem (sic!)» disse un patrizio davanti al conte $\mathrm{e}^{70}$.

Eccessi politici, eccessi verbali: «si dominus comes percuteret me, ego frangerem eius vultum» dichiarò un contadino nel $1424^{71}$; o, il 30 ottobre 1424, un insulto a uno dei giudici locali: «tu non es iudex, imo es vilis pastor animalium et filius unius pastoris» ${ }^{72}$. La forte presenza di violenza retorica, ma anche fisica, i riferimenti quasi quotidiani agli statuti, il ruolo di questi come vero e proprio pomo della discordia tra i curzolani avevano un impatto considerevole sui fratelli.

Da parte veneziana, la posizione dei conti era delicata. Non disponendo di un personale amministrativo o militare imponente, dipendendo dal sostegno dei ceti dominanti locali, erano costretti a navigare in acque torbide, spesso tra due fuochi, perché la dedizione a Venezia non aveva temperato i conflitti locali tra patrizi, cittadini e villici. Questi due fuochi però erano mutabili in funzione delle vicende dei conflitti locali, ed era necessaria una profonda conoscenza della società locale per capire le complicatissime linee di lealtà e inimicizia, una conoscenza che determinava anche le possibilità di mediazione. Infine, non bisogna dimenticare che i Curzolani si confrontavano con una terza dimensione di presenza veneziana: oltre al conte e alle autorità centrali, che rappresentavano lo stato, i patrizi veneziani, spesso di passaggio.

Una situazione insomma che era tipica per i rettori veneziani in molte parti dello stato ${ }^{73}$. Per placare queste tensioni, Venezia non usò bombarde come a Traù (Trogir), ma ricorreva al proprio 'mito'. Nei primi anni del suo dominio su Curzola, dopo le vittorie contro il Duca di Milano la Signoria graziò almeno due volte persone condannate ${ }^{74}$. Il successo militare e la clemenza strategica dovevano impressionare tutti i Curzolani e ricordare loro la potenza politica e militare che,

${ }^{69}$ Ibid., f. 83 r.

${ }^{70}$ Ibid., f. 33v.

${ }^{71}$ Ibid., 6/1/6, f. 3 r.

${ }^{72}$ Ibid., f. 47 r.

${ }^{73}$ O'Connell, Men of Empire, pp. 75ss.

${ }_{74}$ DAZ AK, 6/6/6, ff. 90r, 91r, "cassatum mandato dominorum comitis et iudicum de gratia facta in exultationem gaudii victorie habite [...] contra Ducem Mediolani». 
anche se non visibile sull'isola, seppe punire e ricompensare. Questa politica di grazia completava il sistema di comunicazione istituzionalizzata (petizioni) e di controllo (sindici, avogaria de comun, auditori nuovi) tra periferia e centro.

Sarebbe però erroneo ridurre la presenza veneziana e la socializzazione socio-politica dei Curzolani a un'ininterrotta serie di conflitti e umiliazioni dei conti, all'impressione di un dominio fragile e insicuro. Percorrendo i registri della cancelleria, diventa anzi evidente il buon funzionamento della giustizia che Venezia aveva ereditato a Curzola, la collaborazione tra conte e giudici locali, l'integrazione nelle strutture centrali e centralizzanti del sistema veneziano; e quest'aspetto segnerà in modo indelebile le scelte e il comportamento dei due fratelli. Il mondo dei Curzolani non era condizionato unicamente dal quadro amministrativo veneziano, ma anche soprattutto da diversi livelli di appartenenze locali, appartenenze a varie forme di comunità: quella dell'isola anzitutto che rappresenta, malgrado le tensioni interne, una entità mentale e politica di fronte agli altri, e, almeno nella nostra documentazione esclusivamente veneziana, gli altri per tutti i curzolani, i forenses, erano i vicini della terraferma, prima di tutto i Ragusani, nella logica del campanilismo dalmata nemici di prima classe, poi seguiva la gente della Krajina, cioè dell'entroterra che apparteneva in parte all'Erzegovina, ma anche i pochi italiani, spesso pugliesi o abruzzesi, immigrati nella città portuaria. Questa solidarietà insulare si manifestava nella guardia marittima che, comunque, allo stesso tempo provocava continui conflitti tra patrizi e contadini, gli ultimi rifiutando di prestare troppi servizi. Anche i nostri Dragačići, una volta che avevano fatto carriera, pagavano un supplente.

Quali sono le conclusioni che si possono dedurre dall'esempio microstorico dei Dragačić per le grandi questioni delineate all'inizio del nostro intervento? Prima di tutto occorre constatare che il caso dei Dragačić è probabilmente solo eccezionale a causa della ricchissima documentazione che non si può esaurire in un breve contributo. $\mathrm{Ci}$ sarebbero tanti altri casi di popolani che approfittavano della dedizione a Venezia per far carriera nel nuovo quadro di potere. Forse le nostre osservazioni non forniscono risultati assolutamente nuovi, ma piuttosto elementi che arricchiscono il dibattito: a Curzola, Venezia ereditò una lunga tradizione di auto-amministrazione comunale, una 
rete fitta d'istituzioni in città e nell'entroterra; è probabile che il governo veneto contribuisse ad allargare l'attività della cancelleria, ma il controllo sulle attività economiche e sociali dell'isola era già altissimo quando arrivò il primo conte.

Occorre ricordare prima i limiti delle nostre osservazioni che valgono per il XV secolo, secolo comunque centrale della Venezia medievale e della Dalmazia prima delle incursioni ottomane. Fu davvero un'Altra Venezia quest' isola di Curzola tardo medievale? Malgrado le differenze ovvie, cioè di lingua, l'integrazione dei Dragačić non sarebbe stata possibile senza un'intima familiarità con le regole fondamentali delle istituzioni veneziane, una familiarità con non si limitava ai ceti d'élite. I Dragačić riuscirono a inserirsi nei meccanismi dell'apparato statale veneziano anche - e soprattutto - grazie alla loro socializzazione locale, una tradizione di partecipazione locale, a livello di villaggio, tradizione ad argomentare, a difendere il proprio diritto, una conoscenza degli statuti e delle consuetudini, una familiarità con le procedure giuridiche e soprattutto con la dimensione scritta del diritto. I contadini curzolani difesero tenacemente i loro privilegi come lo facevano gli abitanti delle valli del Bergamasco ${ }^{75}$. Certo, i Curzolani degli anni venti del XV non appartenevano al cerchio interno dei sudditi veneziani come le comunità del dogado, ma un secolo più tardi il discorso di lealtà spiccata è già ben radicato in Dalmazia. Il modello di Altre Venezie vale, con alcune riserve, sicuramente anche per il nostro caso. Quanto a Venezia mediatrice, il caso dei Dragačić ci insegna a distinguere tra le varie dimensioni della statualità veneziana con le quali si confrontarono i sudditi. I Dragačić dovettero il loro successo nel quadro di questo sistema alla loro capacità di servirsi proprio di questa multidimensionalità dello stato veneziano, adattando le alleanze ed aderenze secondo il caso ora alla persona del conte, ora alle autorità centrali, sempre invocando l'immagine della Venezia ideale. I conti servivano certo come intermediari tra sudditi e la dominante, ma furono non raramente anche ostacoli per una pacifica mediazione tra interessi divergenti, agendo ora come soggetti, ora come oggetti della politica locale. Infatti, solo le moltiplici emanazioni della sta-

75 S. Rота, La politica di Venezia nei confronti del territorio bergamasco nel primo secolo di dominazione, in Venezia e Le istituzioni di Terraferma, pp. 67-77. 
tualità veneziana - $\mathrm{i}$ conti, le istituzioni centrali, l'idea dello stato - le sue combinazioni sempre variabili garantivano un equilibrio politico, equilibrio fragile nei primi decenni, ma un equilibrio che si stabilì gradualmente grazie alle forze integrative, forze extraistituzionali come il commercio. Si ammetterà facilmente, viste le vicende dei Dragačić, che i meccanismi statali esercitavano un impatto decisivo sulle pratiche politiche e socio-culturali dei Curzolani. Benché non raramente i conti fossero personalmente deboli, indecisi, la loro posizione personale (non istituzionale!) ambigua o fragile, contestata dagli stessi Curzolani, benché questi cercassero per lungo tempo di strumentalizzare i conti e le istituzioni veneziani ai loro scopi personali, accettarono già dall'inizio norme e regole fondamentali del sistema politico della Serenissima: cioè il primato del diritto, una comunicazione politica sottomessa a strumenti (congregazioni autorizzate, ambasciate a Venezia) e una retorica che valeva in tutto lo stato veneziano. Il potere veneziano non risiedeva unicamente su una strategia di comunicazione, ma su istituzioni stabili e - agli occhi dei governati - affidabili ${ }^{76}$. La statualità veneziana - in Dalmazia e nella Terraferma - era molto più che un sistema retorico; si caratterizza piuttosto come una combinazione riuscita di diritto e istituzioni centrali con il sistema politico-costituzionale locale. La presenza istituzionale del Dominio veneto su Curzola era 'leggera', ma di solito efficace; e quando falliva sul livello locale, le autorità centrali equilibravano la situazione. Le realtà della vita amministrativa e delle interazioni tra le varie emanazioni del governo veneziano e le comunità locali si rivelano più complesse di un'idealizzazione del governo veneziano (secondo il 'mito') o una prospettiva unicamente o prevalentemente negativa (partendo dalla 'storia nera'). Quello che conta è il fatto che dopo decenni di tensione e di vari tipi di conflitto, il sistema si stabilizzò di fronte alla crescente minaccia ottomana. La prima prova seria della lealtà curzolana allo stato veneziano fu però l'invasione di una flotta napoletana che devastò una buona parte dell' isola (1484). Non bisogna sottovalutare i fattori esterni che, come altrove nello stato veneziano, contribuivano

${ }^{76}$ Il caso di un nobile rurale che, nel 1468, accusò il conte di non aver pagato cinque maiali e lo minacciò con le parole «io andarò a Veniexia e lamentarò me de lui» rappresenta solo uno tra tanti esempi (DAZ AK, 20/36/12, f. 14r). 
a creare legami di lealtà. Ma come si è dimostrato in questo saggio i meccanismi interni, politici e amministrativi, rivestono un'importanza maggiore nel processo di integrazione. Questi meccanismi sono da contestualizzare in un ambito adriatico che oltre "pace e giustizia» attirava con le sue possibilità economiche. I due fratelli si arricchirono non solo di prestigio, ma anche di denari guadagnati nel commercio adriatico e nelle attività agricole, una combinazione di honor et proficuum alla maniera (non solo) dalmata.

Diventa evidente anche quanto è problematico parlare di centro e di periferia: Curzola, il primo porto veneziano nella Dalmazia, disponeva di un'ottima comunicazione con la Dominante, i Curzolani interagivano con competenza e talvolta furbizia con le moltiplici dimensioni di statualità veneziana. Venezia mediatrice - certo, ma bisogna tener conto dei limiti della mediazione; neanche le ambasciate a Venezia possono essere considerate come strumento di moderazione; il ritorno di vincitori e vinti, la decisione nelle mani delle autorità centrali non raramente aggravavano la situazione. Nel 1443, il conte costrinse otto uomini, patrizi e popolani, a presentarsi ogni sera in cancelleria «quia [...] contaminabant [...] habitantes in hac insula ad partialitates et odia qui incepti sunt postquam aliqui eorum venerunt de Venetiis» ${ }^{77}$.

Altre Venezie infine - forse non nella stessa misura che nel Dogado, ma, dal punto di vista strutturale, Curzola con la sua lunga tradizione comunale, con la mentalità profondamente marcata di varie possibilità di partecipazione politica, di articolazione di interessi particolari, non differiva troppo da altri comuni che furono integrati nello stato veneziano nel XV. Abbiamo accennato ai casi che illustrano quanto velocemente si ricorreva a strategie identitarie che fusero la propria tradizione comunitaria con il mito di Venezia portatrice di giustizia e di pace. Una lealtà che è il risultato non di un atto spontaneo, ma cresceva lentamente: una crescita alimentata meno dalla presenza fisica, cioè i conti, ma dal funzionamento delle autorità centrali, giuridiche anzitutto. Il caso dei Dragačić dimostra l'enorme potenziale d'integrazione che gli auditori novi possedevano, un'integrazione comunque che era basata sul sapere, sulla conoscenza di questo sistema che precede l'inizio del dominio veneziano.

\footnotetext{
77 DAZ AK, 10/14/1, f. 173r.
} 
Che i Curzolani facessero uso di questo sistema già pochi anni dopo la dedizione, prova la loro intima familiarità con il sistema veneziano. E che questo non fosse un privilegio dei ceti benestanti è illustrato da un ultimo esempio: una famula che aveva ucciso il suo bambino neonato sosteneva di averlo affidato a due donne albanesi che stavano viaggiando a Venezia dove queste avrebbero dovuto consegnare il bambino all' "hospitalis Domus Dei» ${ }^{78}$. Questa povera famula, che si salvò scappando di notte dalle carceri, aveva sentito dell'esistenza di istituzioni caritative ed è l'esempio concreto di quanto era diffusa la conoscenza del sistema politico e giuridico della Serenissima.

Il percorso biografico dei Dragačić, certo non esaurito in questa sede, ci aiuta a comprendere meglio le dimensioni della statualità veneziana su un'isola che non fu una colonia, neanche una periferia lontana, ma che faceva parte del mondo della cultura urbana adriatica che, assieme al Dogado e alla Terraferma, costituiva uno spazio comune di tradizioni comunali ${ }^{79}$. Molte sono le proposte per circoscrivere l'essenza, il carattere della statualità veneziana. Questo saggio ha cercato non solo di offrire una prospettiva microstorica, ma anche di evidenziare il peso della comunità locale. La frammentazione delle società locali sotto il dominio veneziano costituisce il fondamento del sistema di mediazione veneziano: senza conflitti, senza comunità che ricorrevano a meccanismi politico-amministrativi dello stato veneziano, i rettori non avrebbero mai giocato il ruolo di mediatore accettato da tutti i partiti e frazioni locali. Mentre questo rapporto è stato studiato ripetutamente, bisogna approfondire l'analisi della comunità locali. La prospettiva della ricerca spesso riproduce lo sguardo delle nostre fonti, cioè quello delle autorità centrali. Ma l'intreccio dei rapporti tra le varie emanazioni di statualità veneziana e le complessità sociali nelle società e comunità locali richiede una prospettiva più ampia che penetri le dinamiche sociali di quest'ultime. Il caso curzolano dimostra che una rapida e semplificante riduzione delle complesse strutture sociali a un sistema binario patrizi/popolani spiega solo fino a un certo punto il processo di integrazione dell'isola nello stato veneziano. L’opposizione tra le due comunità costituzionali

78 Ibid., 6/6/6, f. 69r.

79 B. Arbel, Régime colonial, colonisation et peuplement: le cas de Chypre sous la domination vénitienne, "Sources travaux historiques», 43-44 (1995), pp. 95-103 distingue tra «colonies de peuplement, colonies d'exploitation et colonies mixtes». 
stava certo alla base di conflitti che strutturavano la società locale e dei quali i rettori erano costretti a tener conto nella prassi amministrativa. Ma il sistema era più flessibile e permetteva a patrizi e popolani alleanze commerciali e talvolta anche politiche. Il caso di Curzola conferma la visione di comunità costituite da un quadro istituzionale, da rapporti sociali e dal potenziale di mobilitazione in momento di conflitto, cioè di visualizzare la comunità come entità sociale nello spazio pubblico ${ }^{80} . \mathrm{Si}$ rivela ugualmente flessibile e sottile il sistema veneziano in cui le autorità centrali equilibrano le attività e le scelte politiche dei rettori, ed era proprio su questa capacità di accomodare tensioni che risiedeva la fiducia degli amministrati. Per il nostro caso dunque il modello di un Dominio a patti tra partner ineguali, come altrove in Dalmazia o nella Terraferma, costituisce un approccio utile per avvicinarsi alle complesse e dinamiche realtà di questa società adriatica tardo medievale nel suo rapporto con la Repubblica di Venezia. Le modalità e le strutture del pensiero politico su Curzola, il potenziale politico e intellettuale dei Curzolani che facilitò una rapida integrazione nelle strutture amministrative e costituzionali dello stato veneto, la capacità di far uso del 'mito' veneziano per una retorica politica molto efficace di fronte ai rappresentanti di questo stato permette di applicare il modello di 'Altre Venezie' anche al caso di Curzola, sottolineando però che nel Quattrocento questo modello si basava non tanto sull'imitazione di strutture veneziane, quanto sull'appartenenza a un mondo adriatico che condivideva certo la stessa storia giuridico-costituzionale, ma che era lontano dal costituire un'entità culturale e linguistica compatta. Il sistema di "pace e giustizia» si sovrapponeva lentamente e finalmente con successo su Curzola, perché fu il sistema che gli stessi Curzolani avevano desiderato, un sistema il cui sviluppo era anche oggetto di negoziazione tra la Capitale e le comunità curzolane. Che questo non fosse il caso in tutta la Dalmazia, è ben noto. Perciò non si devono generalizzare le conseguenze di questo studio di un caso specifico. La Dalmazia offre non uno, ma molti modelli di rapporti statuali tra la Capitale e i suoi possessi territoriali tra Bergamo e Cipro.

${ }^{80}$ Muir, The Idea of Community, p. 10; V. Groebner, Zu einigen Parametern der Sichtbarmachung städtischer Ordnung im späteren Mittelalter, in Stadt und Recht im Mittelalter/La ville et le droit au Moyen Age, a cura di P. Monnet - O.G. Oexle, Göttingen 2003, pp. 133-151. 
